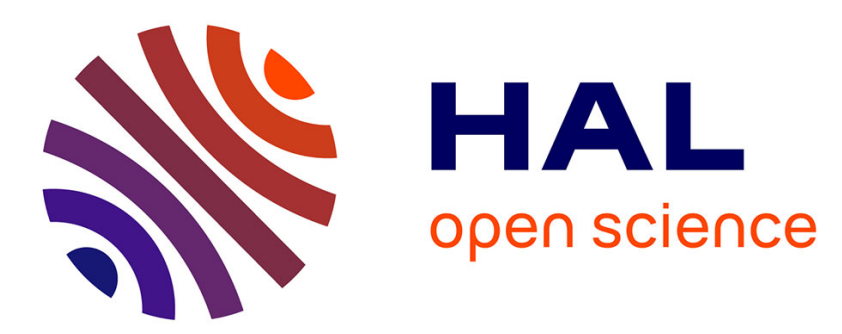

\title{
Influence of thermal diffusion and shear-thinning during the leveling of nanoimprinted patterns in a polystyrene thin film
}

\author{
Hubert Teyssèdre, Stefan Landis, Pierre Gilormini, Gilles Regnier
}

\section{- To cite this version:}

Hubert Teyssèdre, Stefan Landis, Pierre Gilormini, Gilles Regnier. Influence of thermal diffusion and shear-thinning during the leveling of nanoimprinted patterns in a polystyrene thin film. Applied physics. A, Materials science \& processing, 2015, pp.22. 10.1007/s00339-015-9248-3 . hal-01179289

\author{
HAL Id: hal-01179289 \\ https://hal.science/hal-01179289
}

Submitted on 22 Jul 2015

HAL is a multi-disciplinary open access archive for the deposit and dissemination of scientific research documents, whether they are published or not. The documents may come from teaching and research institutions in France or abroad, or from public or private research centers.
L'archive ouverte pluridisciplinaire HAL, est destinée au dépôt et à la diffusion de documents scientifiques de niveau recherche, publiés ou non, émanant des établissements d'enseignement et de recherche français ou étrangers, des laboratoires publics ou privés. 


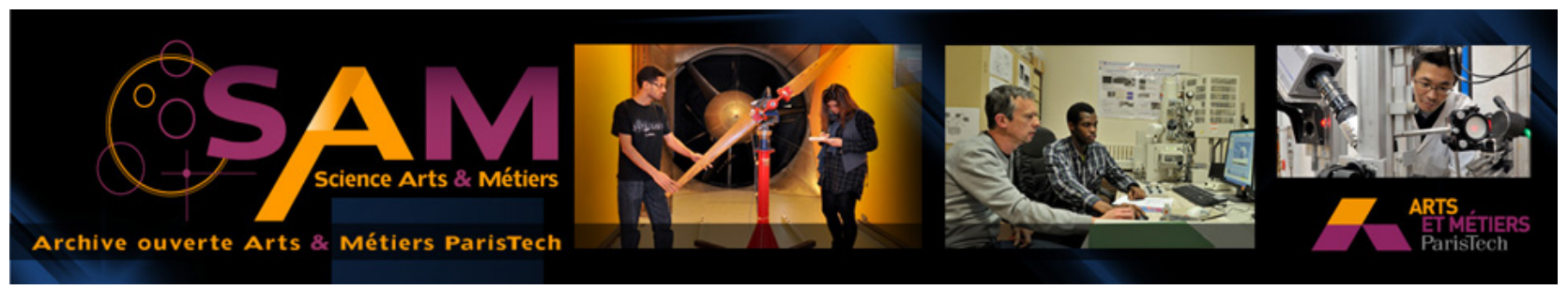

Science Arts \& Métiers (SAM)

is an open access repository that collects the work of Arts et Métiers ParisTech researchers and makes it freely available over the web where possible.

This is an author-deposited version published in: http://sam.ensam.eu

Handle ID: .http://hdl.handle.net/10985/9845

\section{To cite this version :}

Hubert TEYSSĖDRE, Stefan LANDIS, Pierre GILORMINI, Gilles REGNIER - Influence of thermal diffusion and shear-thinning during the leveling of nanoimprinted patterns in a polystyrene thin film - Applied Physics A p.22 - 2015 


\title{
Influence of thermal diffusion and shear-thinning during the leveling of nanoimprinted patterns in a polystyrene thin film
}

\author{
Hubert Teyssèdre ${ }^{1}$, Stefan Landis $^{1}$, Pierre Gilormini $^{2}$, and Gilles Régnier ${ }^{2}$ \\ 1 CEA/LETI, 17 rue des Martyrs, 38054 Grenoble, France \\ hubert.teyssedre@cea.fr \\ 2 PIMM, Arts et Métiers ParisTech, CNRS, CNAM, 151 bvd de l'Hôpital, 75013 \\ Paris, France
}

\begin{abstract}
When capillary forces prevail, the leveling of the free surface of a fluid film is a natural phenomenon that has already found applicative interest either with brushmarks for paint coatings or for rheology on polymeric thin films. Among many parameters, the material behavior influences notably this phenomenon and its characterization still arouses curiosity at the nanoscale. In this article the nanoscale properties of a polystyrene film are derived from the leveling rate of nanoimprinted patterns and are compared to bulk values obtained with a parallel plate rheometer. In particular the focus is made on the isothermal assumption during the process and the consequences of an anisothermal state on the material behavior. Both points are investigated by using numerical simulations based on the natural element method.

First we demonstrate experimentally that the leveling rate is influenced by the heat exchange at the air-polymer interface and that thermal diffusion should be taken into account within the film and its underlying substrate. Then we numerically investigate the influence of thermal diffusion and shear-thinning on the leveling rate. Finally we show that the bulk properties can represent particularly closely the behavior of the polymer at the nanoscale if adequate thermal boundary conditions are used and if shear-thinning is taken into account. This agreement postulates a decrease by $7^{\circ} \mathrm{C}$ of the mean temperature of the polystyrene film coated on silicon when experiments are carried out on a hotplate at $100^{\circ} \mathrm{C}$ in a cleanroom environment.
\end{abstract}

Keywords: Leveling, nanoimprint, shear-thinning, thermo-mechanical simulation, polystyrene

\section{Introduction}

Nanoimprint is a cost efficient technique developed by Chou et al. [1] to replicate nanoscale patterns by embossing a stamp in a polymeric thin film coated on a substrate. Behind this elementary process, a collection of progresses and developments has been acquired over the last 20 years, like complex and large scale stamp 
manufacturing processes [2], anti-sticking layer optimization [3] and characterization [4], dedicated imprint materials, dedicated metrology approaches [5] and commercial imprint equipment allowing high resolution and large scale patterning solutions. Thanks to that, tremendous improvements were achieved for process know-how and optimization, making feasible the assessment of NanoImprint Lithography (NIL) technology for a wide range of applications [6-9]. Although the technology principles seem simple compared to more sophisticated and wellestablished pattern technologies (Extreme Ultraviolet Lithography, $193 \mathrm{~nm}$ immersion lithography, multibeam electron lithography) its implementation to high or even medium scale volume production remains challenging and a complete available process chain (equipment, material, processes, metrology and simulation) is still missing to accelerate the NIL adoption in manufacturing environments. Despite the NIL process simulation gained maturity over the last 10 years it remains the weakest link of the full NIL process chain. At first sight, NIL processes involve multidisciplinary concepts (fluidics, chemistry, mechanics, thermal considerations, surface science) that are not yet fully integrated in a single and validated simulation tool. In the second place, the dedicated and available tools, like the one proposed by Schulz et al. [10] based on nanoimprint experiments with polystyrene, have a limited range of applications. The main reason is that the mechanical behavior of the thin and ultra-thin polymeric films, which is considered as input of simulation tool, are not yet well-established. The development of turnkey solutions to simulate the process will be undoubtedly accompanied by improvements on the mechanical characterization of thin films. To date, this characterization still raises unsolved questions: does the bulk properties apply at the nanoscale? How to explain or model the transition between these two scales? How relevant material properties at the nanoscale can be obtained? Keddie et al. brought a part of the answers by studying the thickness dependence of the glass-transition temperature $\left(T_{g}\right)$ on both polystyrene [11] and poly(methyl methacrylate) [12]. These authors highlighted the existence of a critical thickness, that depends on the material and the underlying substrate, below which $T_{g}$ varies. These results are valuable for modeling and simulating any forming process which deals with micro- and nanoscale polymer flows, even if some discrepancies stand between the different experimental methods [13]. Similar conclusions have been ventured in films of tens of nanometers for the other dynamic properties, such as the polymer chain diffusion [14], the viscosity [15] or the relaxation time [16]. As these measurements are linked with an analytic model, one question can be raised: to what extent can these nanoscale effects on the dynamic properties be trusted?

The leveling process, which is the focus of this paper, is one method that allows deriving the nanoscale material properties of a fluid film by measuring the leveling rate of a corrugation on its free surface. This method has been applied to polystyrene (PS) by Leveder et al. [17] and Rognin et al. [18,19], and to poly(methyl methacrylate) (PMMA) by Jones et al. [20] and Teisseire et al. [21]. The polymer films are patterned with the thermal nanoimprint process in order to preserve the physical and chemical properties of the polymers. In 
this method the derivation step is a critical point as it involves an underlying modelization of the polymer flow during the leveling. This model is usually built with simplifying assumptions to be able to compute an analytic solution, but this brings restrictions to the measurements range inevitably. These limitations have been investigated numerically by Gilormini and Teyssèdre [22] for Newtonian viscous behaviors with the assumption of a uniform temperature within the film. The viscous behavior and isothermal hypotheses are examined in this paper on a periodic square wave profile with a wavelength of $2 \mu \mathrm{m}$ and an initial height of $126 \mathrm{~nm}$.

The remainder of the paper is organized as follows. Section 2 presents the equipments, as well as the polymer used and more precisely its bulk behavior. Section 3 introduces the leveling process and the experimental results demonstrating that improved models have to be considered to well describe the polymer reflow. Section 4 extends the code already used in a previous publication [22] to non isothermal flows and shows the impact of thermal diffusion and shearthinning on the leveling process for a Newtonian behavior. The additional effect of shear-thinning is developed in section 5 where the relevance of the leveling method and the material parameters derivation are also discussed.

\section{Equipments and material}

\subsection{Equipments}

A parallel plate rheometer was used to obtain the linear viscoelastic properties of the bulk polymer. The measurements were performed on $25 \mathrm{~mm}$ round plates at discrete temperatures over the glass transition temperature $T_{g}$. A frequency sweep, from $10^{-2}$ to $10^{2} \mathrm{~s}^{-1}$ was imposed to the sample keeping the deformation amplitude below $10 \%$. Viscous behavior versus shear rate is obtained according to the Cox-Merz principle [23].

For thin film characterization, diluted polymers were spin coated on the $200 \mathrm{~mm}$ silicon substrates. The annealing step (post bake to evaporate the residual solvent) and leveling process (also called reflow step) were performed on the hot plate where the temperature is controlled with an error of $\pm 0.1^{\circ} \mathrm{C}$ and where the substrate is hold with vacuum holes to ensure a perfect thermal contact.

The resist patterning processes were performed using a NIL set-up from EVG (EVG520HE) with a silicon hard stamp. The design of the stamp contains arrays of lines with a square wave profile having a pitch of $2 \mu \mathrm{m}$ and an initial height of $126 \mathrm{~nm}$. In all cases the demolding was performed manually at room temperature.

The free surface characterizations (topography profile measurements) were performed with an Atomic Force Microscope (AFM) Veeco 3000A for the initial printed pattern and after all the successive annealing steps (every $30 \mathrm{~s}$ ). High aspect ratio tips were used, with a radius of curvature of $15 \mathrm{~nm}$ and a half cone angle of $5^{\circ}$. 


\subsection{Material and bulk properties}

A commercial plastified polystyrene from Aldrich (331651) with a molecular mass $M_{w}=35 \mathrm{~kg} / \mathrm{mol}$ (PS35) is used for the experiments. The glass transition temperature $T_{g}$ has been measured on a TA instruments DSC Q10 and found to be close to $60^{\circ} \mathrm{C}$, namely $40^{\circ} \mathrm{C}$ lower than the usual $T_{g}$ for polystyrene of $35 \mathrm{~kg} / \mathrm{mol}$ in molar mass. This difference is lightly due to the presence of the platicizer. Its mechanical characterization, presented in figure 1 at 90, 100, 110 $120^{\circ} \mathrm{C}$, exhibits a Newtonian behavior at low shear rates with shear-thinning at high shear rates for a given temperature. A continuous description is made for a selected temperature $T_{j}$ with the Carreau-Yasuda law [23]

$$
\eta(\dot{\gamma})=\frac{\eta_{j}}{\left(1+\left(\dot{\gamma} / \dot{\gamma}_{j}\right)^{k}\right)^{\frac{1-n}{k}}}
$$

where $\eta_{j}$ is the zero shear rate viscosity (Newtonian plateau), $\dot{\gamma}_{j}$ the critical shear rate, $n$ the pseudo-plastic exponent and $k$ being a fitting parameter. Besides, $\eta_{j}$ and $\dot{\gamma}_{j}$ follow the Williams-Landel-Ferry (WLF) law [25]. The measurements at the defined temperature $T_{j}$ are thus linked by

$$
\log \frac{\eta_{j}}{\eta_{s}}=\log \frac{\dot{\gamma}_{s}}{\dot{\gamma}_{j}}=\log a\left(T_{j}\right)=-C_{1} \cdot \frac{T_{j}-T_{s}}{T_{j}-T_{\infty}},
$$

where $C_{1}, T_{\infty}$ and $T_{s}$ are the WLF coefficients, $\eta_{s}$ and $\dot{\gamma}_{s}$ being defined at the reference temperature $T_{s}$.

$T_{s}=T_{g}+50=110^{\circ} \mathrm{C}$ is fixed according to the rules of [25] and the remaining parameters values are derived from our measurements, with the best fit obtained for $C_{1}=6.8, T_{\infty}=22^{\circ} \mathrm{C}, \eta_{s}=12.5 \mathrm{kPa} . \mathrm{s}, \dot{\gamma}_{s}=0.07 \mathrm{~s}^{-1}, k=0.98$ and $n=0.54$.

The pending drop method is used to measure the surface tension $\sigma=40 \mathrm{mN} / \mathrm{m}$, found to be almost constant in the range of 90 to $160^{\circ} \mathrm{C}$. This value is higher than the ones reported by Bicerano [26] which are between 30 and $35 \mathrm{mN} / \mathrm{m}$ for pure polystyrene. Like the low value of $T_{g}$ the value of $40 \mathrm{mN} / \mathrm{m}$ may be explained by the presence of additives in the material. This value is then kept for our simulations.

\section{$3 \quad$ Experiments}

During the leveling process the polymer temperature is usually assumed to be constant through the film and substrate thicknesses. As seen in figure 1 the temperature strongly impacts the polymer viscosity and is therefore a critical parameter for the process. In this section a set of experiments is conducted to revisit the isothermal assumption by investigating the leveling of periodic gratings of PS35. These experiments will help us to quantify the effect of the heat transfer between the polymer film and the air on the leveling rate. 


\subsection{Procedure and samples}

A dilution of $6 \%$ in mass of PS35 in isopropanol is deposited on a $200 \mathrm{~mm}$ silicon wafer, then spin coated to obtain a film with a mean thickness of $126 \mathrm{~nm}$. An annealing step is performed during $120 \mathrm{~s}$ at $T_{g}+40^{\circ} \mathrm{C}$ to remove the residual solvent within the film. Then parallel lines are printed in the film at $100^{\circ} \mathrm{C}$ for 2 hours to erase any residual stresses due to the polymer flow as pointed out by Ro et al. [27] and Papenheim et al. [28]. The wafer is then sliced into samples of $2 \times 2 \mathrm{~cm}$ and only 4 samples are retained to ensure a polymer thickness variation lower than $1 \mathrm{~nm}$ between one another. This procedure allows us to minimize any geometrical and material composition variations.

The main steps of our method of leveling are illustrated in figure 2. After being imprinted the profile is measured by AFM to extract the initial amplitude and pitch of the profile. The film is then transferred to a hotplate held at a definite temperature above $T_{g}$ during a definite time. Then the film is rapidly cooled down and the reflowed profile is measured by AFM again, with an error of $\pm 2 \%$. The two latter steps are repeated until the amplitude reaches a few nanometers in order to record the evolution of the amplitude versus the annealing time. As it has been already shown by Leveder et al. [17], the square wave profile is progressively transformed into a sinusoidal one, which corresponds to the main mode of the initial profile in the Fourier series, and finally to a flat surface.

Two set of experiments were performed on the hotplate at $100^{\circ} \mathrm{C}\left(T_{g}+40^{\circ} \mathrm{C}\right)$. The first one, referred as the Open Ball configuration, consists in leaving two samples on the hotplate and measuring the profile by AFM every $30 \pm 1 \mathrm{~s}$ with the samples directly exposed to the laminar air flow of the clean room thermalized at $25^{\circ} \mathrm{C}$. The second experiment follows the same procedure but with a lid over the two other samples to prevent any convection flux around the sample and to reduce the heat transfer. The latter case is named Lid On configuration.

An AFM measurement is performed after each period after quenching the sample on a cold aluminum plate.

\section{$3.2 \quad$ Experimental results}

The evolution of the gratings amplitudes during the leveling process is presented in figure 3, for both configurations, the Open Ball one (crosses) and the Lid On one (filled circles). First we notice that, in both cases, the experiments are quite repeatable and that two trends are clearly emerging. The amplitudes of the reflowed structures in the Lid On configuration are deceasing more rapidly than the ones in the Open Ball case. As both set of experiments were carried out with the same material, same resist thickness, same initial printed pattern on the same wafer, it appears that thermal considerations within the polymer film and heat exchanges with the environment may be the cause of such differences. In the Lid On configuration the sample is isolated from the laminar air flow of the clean room. The lid we use leaves a layer of air of arround $1 \mathrm{~cm}$ over the sample, covers the whole hot plate, even on the edges, and provides a thermal isolation from the ambient air. The temperatures of the film and the enclosed 
air are then expected to be close to the hotplate temperature. In the Open Ball case the ambient air tends to cool the free surface of the film, which may imply a temperature gradient in the film thickness.

The main difference between the two cases stands in the thermal inertia of the air above the samples when the lid is down. If the air close to the free surface has quickly a temperature close to the hotplate one, then the difference with and without the lid may be imperceptible. On an indicative basis we put a thermal sensor at around $5 \mathrm{~mm}$ over the plate. In the Open Lid case after $2 \mathrm{~min}$ the temperature was stabilized and was between 28.9 and $30.5^{\circ} \mathrm{C}$. This range was the same after $5 \mathrm{~min}$. Then we closed the lid and measured the temperature every $15 \mathrm{~s}$ for $60 \mathrm{~s}$. We respectively recorded $31.6,35.5,38.4$ and $41.1^{\circ} \mathrm{C}$. Thus the air is expected to be not thermalized during our experiment, at least few millimeters away from the sample. Closer to the sample the temperature should evolve faster and we will assume in the following that a quasi-static thermal state is reached for the air and the polymer close to the free surface.

Our results suggest that the stationary thermal state of the thin films are influenced by the heat exchanges with its environment. This result has a direct impact on the use of the leveling method to estimate the material properties, as discussed in the following subsection.

\subsection{Discussion}

An initial examination of the mean viscosity of the film can be performed by using the method of Leveder et al. [17]. This method consists in evaluating the characteristic decreasing time $\tau$ of the pattern amplitudes and computing the viscosity with the following equation

$$
\tau=3 \eta_{0}(w /(2 \pi))^{4}\left(\sigma \tilde{h}^{3}\right),
$$

where $w$ is the pitch of the pattern, $\eta_{0}$ the polymer viscosity (assumed to be constant), $\sigma$ the surface energy of the polymer-air interface, and $\tilde{h}$ the mean thickness of the film. This formula assumes that the amplitudes are small compared to $w$ and $\tilde{h}$. To fulfill this assumption we focus on the amplitude decays at long annealing times $(>80 \mathrm{~s})$ where the free surface profile is expected to be close to a sinus, and consider the mean slopes plotted in figure 3 . The characteristic decreasing time are respectively $\tau_{O B}=207 \mathrm{~s}$ for the Open Ball case and $\tau_{L O}=130 \mathrm{~s}$ for the Lid On one. The corresponding mean viscosities are then respectively equal to $\tilde{\eta}_{O B}=538 \mathrm{kPa} . \mathrm{s}$ and to $\tilde{\eta}_{L O}=337 \mathrm{kPa} . \mathrm{s}$.

It is worth noting that both $\tilde{\eta}_{L O}$ and $\tilde{\eta}_{O B}$ are higher than the bulk viscosity at low shear rate at $100^{\circ} \mathrm{C}(\eta \approx 80 \mathrm{kPa} . \mathrm{s})$. Two conclusions are then possible: either the temperature within the PS35 was not equal to $100^{\circ} \mathrm{C}$ during the time of experiment or the material does not follow the bulk behavior for a film thickness of $126 \mathrm{~nm}$. The latter conclusion is the one of Teisseire et al. [21] but is debatable if we look at the conclusions of Keddie et al. [11] or, respectively, those of Rowland et al. [29] who found a nanoscale effect below $40 \mathrm{~nm}$, or respectively $10 \mathrm{~nm}$, for polystyrene films. If we assume that the bulk behavior applies to 
our film, then the increase of viscosity is equivalent to a diminution of the PS35 film temperature of $5.3^{\circ} \mathrm{C}$ for the Lid On case and $7.0^{\circ} \mathrm{C}$ for the Open Ball configuration. Here we see that the leveling method combined with equation (3) provides an estimation of the absolute value of the film viscosity but with an uncertainty on temperature.

Regarding these results, it appears that a fine thermal analysis of the leveling process is required to ensure that this approach can be used to extract the mechanical properties of the polymer without any doubt. The thermo-mechanical approach suggested here also points out that simple analytic models are no longer relevant to assess our material properties. A numerical approach is therefore needed and is described in the next section.

\section{Numerical simulations of leveling with thermal diffusion in a Newtonian flow}

The simulation code used in this work applies the natural element method (NEM), which is a natural neighbor Galerkin method that has already been applied to fluid dynamics by Martínez et al. [30] and González et al. [31], for instance. The code includes surface tension and wetting defined by the static contact angle [32]. Newtonian and shear-thinning behaviors can be taken into account. The variational formulation is detailed in [32] and allows to compute the velocity field $\boldsymbol{v}$ and pressure field $p$ in the polymer. The substrate is modelled as a rigid body and is supposed to be fixed. A no-slip condition is considered at the solid-fluid interface. For this paper the code used in [32] has been extended to laminar flows with stationary thermal diffusion.

\subsection{Thermal diffusion modelization}

The thermal inertia of the stack polymer-substrate is neglected as the silicon is supposed to have a low thermal inertia and as the thicknesses of the polymer films are of a few hundreds of nanometers only. This assumption has been investigated by Rognin et al. [18] who demonstrated that the polymer film temperature reaches a stationary state in less than $1 \mathrm{~s}$, which is negligible compared to our leveling times $(\approx 30 \mathrm{~s})$. This assumption is therefore also used in this work but without straightly assuming that the temperature of the polymer film and the substrate are constant and equal to the hotplate temperature. Actually the thermal equilibrium state is mainly ruled by the thermal diffusion equation which writes:

$$
\lambda \Delta T=0 \quad \text { within the materials, }
$$

where $\lambda$ is the thermal conductivity of the considered material and $\Delta$ denotes the Laplacian operator. Equation (4) is completed by thermal boundary conditions, such as imposed temperatures. We assume that the temperature of the polymer at the free surface is constant and is used as an adjustable variable to match the experiments. Then we assume that a temperature $T_{0}$ applies at the interface with 
the substrate, as presented in figure 4, but which is not directly the temperature of the hot plate $T_{H P}$ as it takes into account the thermal diffusion within the silicon substrate. By solving the diffusion equation (4) with the mean thickness $\tilde{h}$ of the film, the temperature $T_{0}$ can be approximated by:

$$
T_{0}=\frac{\frac{\lambda_{S}}{e_{S}} T_{H P}+\frac{\lambda_{P S}}{\tilde{h}} T_{i}}{\frac{\lambda_{S}}{e_{S}}+\frac{\lambda_{P S}}{\tilde{h}}}
$$

where $\lambda_{S}, \lambda_{P S}$ are respectively the thermal conductivity of the silicon and the polystyrene, and $e_{S}$ stands for the thickness of the silicon substrate. $T_{0}$ is thus imposed through $T_{i}$ using the bulk values reported in table 1 . These values are chosen as guides to perform our demonstration on the existence of thermal diffusion. Last we prescribe that the heat flux normal to the symmetry axis is equal to zero.

In our approach the thermal and flow problems are uncoupled and the variation of the temperature with time is neglected in the thermal equilibrium. The temperature field within the material is computed in a first loop and the solution is used to determine the WLF parameter $a(T)$ of equation (2). A second loop that uses $a(T)$ is performed to compute the velocity field that helps to update the nodes positions. After updating the two loops are repeated until the simulated time reaches $240 \mathrm{~s}$.

\begin{tabular}{|c|c|c|c|c|c|}
\hline Parameter & $h$ & $\lambda_{P S}$ & $e_{S}$ & $\lambda_{S}$ & $T_{H P}$ \\
\hline Value & $126 \mathrm{~nm}$ & $0.13 \mathrm{~W} / \mathrm{m} / \mathrm{K}$ & $1.5 \mathrm{~mm}$ & $148 \mathrm{~W} / \mathrm{m} / \mathrm{K}$ & $100^{\circ} \mathrm{C}$ \\
\hline
\end{tabular}

Table 1. Numerical values used for the thermal problem.

\subsection{Equivalent relaxing time}

The focus is made on the decreasing rate of the amplitude of free surface profile. Assuming that the decrease of the amplitude of a sinusoidal profile writes

$$
A(t)=A_{0} \cdot \exp (-t / \tau),
$$

where $A_{0}$ is the initial amplitude, we define $\tau$ which is the relaxing time that characterizes the decreasing rate. This assumption is relevant if the amplitude remains small enough compared to the thickness of the film and the wavelength of the sinusoidal profile. Quantitative rules have been established by Gilormini and Teyssèdre [22] who also demonstrated that, as the amplitude is reduced during the leveling, their rules are fulfilled after a certain time. As $\tau$ is a parameter of equation (6) that can be deduced from experiments and that helped $[17,18,21$, $20]$ to derive the physical properties of the materials, we focus on the quantity

$$
\tau_{e q}(t)=\left(\frac{d \log (A)}{d t}(t)\right)^{-1}
$$


which is calculated on the amplitude of the transient profile that is not necessarily a sinus and therefore $\tau_{e q}$ not necessarily a constant. When $\tau_{e q}$ will reach a constant value, this equivalent time will help us to easily see when the amplitude decrease fits an exponential law. Furthermore it will be an indicator to compare the simulations results to the slopes of the experimental curves.

\subsection{Temperature effect}

In the following we concentrate on the effect of the temperature gradient on the leveling rate of a sinusoidal profile. The critical shear rate $\dot{\gamma}_{s}$ is set to infinity to simulate a Newtonian behavior. The temperature of the hotplate $T_{H P}$ is fixed to $100^{\circ} \mathrm{C}$ and the temperature of the free surface $T_{i}$ varies from 100 to $91^{\circ} \mathrm{C}$. The results are plotted in figure 5 . When $T_{i}=T_{H P}$ the temperature is homogeneous within the film and two regimes can be clearly identified, with a transition at $79 \mathrm{~s}$. In the early stage $\tau_{e q}$ is not constant and increases abruptly up to $75 \mathrm{~s}$ before decreasing slowly down to $47 \mathrm{~s}$ which is its asymptotic value $\left(\tau_{e q}^{\infty}\right)$. The detailed study of $\tau_{e q}$ variations is beyond this work. Nevertheless we can briefly point out that a squeeze effect is encountered where the film has a minimum thickness. This results in a locking effect in this region and a leveling rate which does not follow an exponential law as depicted by the indicator $\tau_{e q}$.

The asymptotic value $\tau_{e q}^{\infty}$ tends to increase as the temperature $T_{i}$ is reduced as it can be seen in figure 5 . This is consistent with the material behavior which tends to be more viscous in the vicinity of the free surface and tends to reduce the leveling rate and therefore increase the leveling time. In the mean time the transitions between the two regimes are also shifted and are respectively located at 135 and $235 \mathrm{~s}$ for 97 and $94^{\circ} \mathrm{C}$. The transition for $91^{\circ} \mathrm{C}$ cannot be plotted in the figure but is larger than $240 \mathrm{~s}$. It is worth noting that even with a non uniform temperature a plateau is obtained for long leveling times, which corresponds to an exponential decrease. The gradient of temperature (lower than 2 degrees according to equation (5)) in the film thickness has then a small influence on the decreasing mode of the profile. We note that a decrease of the temperature by $1{ }^{\circ} \mathrm{C}$ in the polymer increasing the viscosity by $25 \%$ at $95^{\circ} \mathrm{C}$ and $22 \%$ at $100^{\circ} \mathrm{C}$. This difference impacts directly the decreasing rate. Experimentally this decrease can be easily mistaken with one obtained at a constant temperature, but deriving the material viscosity in this case would induce errors on the temperature of measurement as the temperature decrease within the substrate is much larger (up to 9 degrees).

\section{4 $T_{i}$ adjustment}

Using figure 6 the free surface temperature $T_{i}$ is tuned in order to fit the experimental equivalent relaxing times estimated in section 3.3. In the Open Ball configuration the relaxing time $\tau_{O B}=207 \mathrm{~s}$ is well recovered with $T_{i}=93.7^{\circ} \mathrm{C}$. In this condition the interface between the polymer and the substrate $T_{0}$ is equal to $94.3^{\circ} \mathrm{C}$, which gives us a mean temperature of $94^{\circ} \mathrm{C}$ within the material, which 
is slightly above the mean temperature $\left(93^{\circ} \mathrm{C}\right)$ estimated in section 3.3 when using the analytic solutions. This difference is due to the non linear dependence of the viscosity versus the temperature and is expected to increase for a thinner film. The same conclusions stands for the Lid On case.

The readjustment of the final slope of the amplitude decrease gives nonetheless a bad agreement for the absolute value of the amplitude, as presented in figure 7 . Indeed the short times amplitude variations are not captured as the fitting is performed at long times. In figure 7 the simulations for $T_{i}=93.7^{\circ} \mathrm{C}$ are presented for two initial profiles. A square wave profile (continuous line) with an initial amplitude of $A_{0}=61.4 \mathrm{~nm}$ and a sinusoidal one (dashed line) which is the main Fourier mode of the square wave with an initial amplitude of $4 A_{0} / \pi=78.2 \mathrm{~nm}$. The first one is the most representative of the experimental geometry. An increase of the amplitude at the early stages is first observed, before an exponential decrease of the amplitude. The gap of nearly $11 \mathrm{~nm}$ between the experimental data and the computed one is created as soon as the leveling is initiated. Whatever the leveling time, the simulation is not able to match the experimental measurements. The sinusoidal profile is of interest as it shows that the leveling is globally ruled by the main Fourier mode of the profile and that a better matching would be possible if this main mode was expected to decrease faster in the early stages without altering its final slope. Actually the maximum shear rate $\dot{\gamma}^{\text {max }}$ computed during the leveling of the main mode is found to be constantly larger than the critical shear rate $\dot{\gamma}_{c}$ for $t<100 \mathrm{~s}$, as presented in figure 8. $\dot{\gamma}_{c}=\dot{\gamma}_{s} / a(T)$ as computed with the WLF law (eq. 2) is equal to $2.2 \times 10^{-3} \mathrm{~s}^{-1}$ at $94^{\circ} \mathrm{C}$ and is plotted in continuous line in figure 8 . Therefore shear-thinning is expected to occur and its impact is investigated in the next section.

\section{Shear-thinning and initial profile effects on the anisothermal leveling}

\subsection{Shear-thinning effect}

The shear rates in the previous simulations were found higher than the critical shear rate $\dot{\gamma}_{c}$ which suggests that shear-thinning must occur. This observation is in good agreement with the rule of [22] on the generalized shear rate, which estimates the critical amplitude (below which shear-thinning is not expected) $A_{c}=0.046 \eta_{s} \dot{\gamma}_{s} w^{2} / \sigma \approx 4 \mathrm{~nm}$ with our material and pattern, where $w$ denotes the wavelength of the pattern. As our initial amplitude $(63 \mathrm{~nm})$ is higher than $A_{c}$, shear-thinning has to be considered. Then the previous simulations are rerunned with the shear-thinning behavior. The results are presented in figure 9 in filled symbols. The Newtonian results are presented for comparison, in open symbols, but only for $T_{i}=100$ and $91^{\circ} \mathrm{C}$. We first observe that the evolution of $\tau_{e q}$ has drastically changed. The maximum value is not obtained in the first regime but is found to be the asymptotic value of the second regime. Thus the curves are always below the asymptotic value unlike the Newtonian simulations. The 
transition values between the two regimes has also increased. It can be estimated around $120 \mathrm{~s}$ for $T_{i}=100^{\circ} \mathrm{C}$, around $200 \mathrm{~s}$ at $97^{\circ} \mathrm{C}$ and larger than $240 \mathrm{~s}$ for $T_{i} \leq 97^{\circ} \mathrm{C}$.

Figure 9 also demonstrates that the equivalent relaxing time does not depend on the behavior for the long process time. This can be seen for $T_{i}=100^{\circ} \mathrm{C}$ where the asymptotic values have a negligible difference. For $T_{i}=91^{\circ} \mathrm{C}$ the asymptotic values are not presented but the simulations showed similar results. Hence the derivation of the material properties from the asymptotic values is theoretically possible even if shear-thinning occurs. Nevertheless one pitfall could be the smallness of the amplitude.

\subsection{Initial profile influence}

With nanoimprint technology square wave profiles are more typically produced than sinusoidal corrugations. Thus we compare the leveling rate of these two types of profiles. This comparison is permitted if one considers the Fourier series of the square wave profile and sees it as a sum of sinusoidal profiles. This is only fully relevant with a linear approach but still gives directions with the non-linear one. The amplitude of the initial sinusoidal profile is chosen such as to have the same main mode as the square wave one. As the linear theory predicts that the modes of the square wave profile associated with the short wavelengths vanish almost instantaneously compared to the main one, this phenomenon should be even faster if shear-thinning occurs, since viscosity is locally lower. This assumption is at least validated in our case, as presented in figure 10. In this figure the equivalent relaxing times for an initial square wave profile are presented in filled symbols. The two extreme temperatures $\left(91\right.$ and $\left.100^{\circ} \mathrm{C}\right)$ with the initial sinusoidal profile are also reported in open symbols to ease comparison. Except at the very first stages the equivalent relaxing times are really close regardless the initial profile. The study can consequently be simplified and limited to the investigation of the leveling of the main mode of the profile.

\subsection{Data fitting}

The shear-thinning behavior allows us to fit quite properly the leveling experimental data. Here the fitting is not based on an adjustment of the limit value of the amplitude slope because the plateau is not reached in the simulations for $T_{i}<95^{\circ} \mathrm{C}$. In fact an iterative approach is used with the sinusoidal initial profiles, which is much faster to simulate. The fit is performed without the first experimental data point $(t=0)$. Finally, the simulations with the optimized temperatures and with an initial square wave profile are plotted in figure 11 for both thermal conditions.

In the Open Ball case the same temperature $T_{i}=91.8^{\circ} \mathrm{C}$ as the one of section 4.4 provides the best agreement. The gap described in section 4.4 no longer exists thanks to the addition of shear-thinning as deduced from the bulk measurements. The same conclusions stand for the Lid On configuration with an optimized free surface temperature $T_{i}=93.1^{\circ} \mathrm{C}$. 


\subsection{Discussion}

The adjustment of the simulations has been performed with a single parameter, $T_{i}$, and by using a Carreau-Yasuda law combined with the WLF law for the material behavior. As the optimizations are obtained with really precise temperatures $\left( \pm 0.1^{\circ} \mathrm{C}\right)$, the parameters extracted from the bulk measurements have to be obtained with an equivalent precision on temperature. In our case the uncertainty was closer to $\pm 0.5^{\circ} \mathrm{C}$ on the parallel plate rheometer. This could explain the quite low values obtained for $T_{i}$ compared to the hot plate temperature. A better refinement on the 7 parameters of the two laws is not possible yet because the leveling method suffers from its sensitivity to temperature and the heat exchange between the film and the environment, and its repeatability is still too limited to adjust such complex laws. Nonetheless the method remains reliable to measure the mean viscosity of the films if it is assumed to be uniform in the material.

\section{Conclusion}

The assumption of a constant temperature, equal to the hotplate temperature, in a film during the leveling process has been revisited. A prior experiment has been presented in order to emphasize the influence of the thermal boundary conditions on the leveling rate from which the material properties can be extracted. Taking thermal diffusion into account and using the bulk behavior, numerical simulations have been performed and the results helped us to understand separately the influences of temperature, shear-thinning and the initial shape of the free surface on the leveling rate. Temperature has a strong influence on the limit slope of the amplitude decrease when shear-thinning prevails at the early stages of leveling in the cases considered in this study. The influence of a thermal gradient through the film has been investigated numerically but could not be captured on the experimental data because of the noise in the measurements.

A good agreement has been reached between the simulations and the experiments by tuning the free surface temperature with the combined Carreau-Yasuda and WLF laws. The two optimized values differ by only $1.3^{\circ} \mathrm{C}$, which demonstrates the performance of the method. Nevertheless uncertainties remain about the difference of nearly $7^{\circ} \mathrm{C}$ found between the free surface of the polymer and the hot plate temperature. This difference might be an artifact due to uncertainties in the behavior law parameters that influence the fitting procedure. It could also be due to a temperature gradient that does not start at the interface between the substrate and the hot plate but deeper in the hot plate. Further work should be performed to investigate these possibilities.

\section{References}

1. Chou, S. Y., Krauss, P. R., Renstrom, P. J.: Imprint of sub-25 nm vias and trenches in polymers. Applied Physics Letters. 67, 3114 (1995) 
2. Landis, S., Reboud, V., Enot, T., Vizioz, C.: Three dimensional on $300 \mathrm{~mm}$ wafer scale nano imprint lithography processes. Microelectronic Engineering. 110, 198$203(2013)$

3. Schift, H., Saxer, S., Park, S., Padeste, C., Pieles, U., Gobrecht, J.: Controlled co-evaporation of silanes for nanoimprint stamps. Nanotechnology. 16, S171-S175 (2005)

4. Landis, S., Chaix, N., Gourgon, C., Leveder, T.: Quantitative characterizations of a nanopatterned bonded wafer: Force determination for nanoimprint lithography stamp removal. Nanotechnology. 19, 125305 (2008)

5. Yu, Z., Gao, H., Chou, S. Y.: In situ real time process characterization in nanoimprint lithography using time-resolved diffractive scatterometry. Applied Physics Letters. 85, 4166-4168 (2004)

6. Schift, H.: Nanoimprint lithography: An old story in modern times? A review. Journal of Vacuum Science and Technology B: Microelectronics and Nanometer Structures.26, 458-480 (2008)

7. Truskett, V. N., Watts, M. P. C.: Trends in imprint lithography for biological applications. Trends in Biotechnology. 24, 312-317 (2006)

8. Yu, C.-C., Chen, H.-L.: Nanoimprint technology for patterning functional materials and its applications. Microelectronic Engineering. 132, 98-119 (2015)

9. Kooy, N., Mohamed, K., Pin, L. T., Guan, O. S.: A review of roll-to-roll nanoimprint lithography. Nanoscale Research Letters. 9, 1-13 (2014)

10. Schultz, H., Wissen, M., Bogdanski, N., Scheer, H. C., Mattes, K., Friedrich, Ch.: Impact of molecular weight of polymers and shear rate effects for nanoimprint lithography. Microelectronic Engineering. 83, 259-280 (2006)

11. Keddie, J. L., Jones, R. A. L., Corry, R. A.: Size-dependent depression of the glass transition temperature in polymer films. Europhysics Letters. 27, 59 (1994)

12. Keddie, J. L., Jones, R. A. L., Corry, R. A.: Interface and surface effects on the glass-transition temperature in thin polymer films. Faraday Discussion. 98, 219 $230(1994)$

13. Alcoutlabi, M., McKenna, G. B.: Effects of confinement on material behaviour at the nanometre size scale. Journal of Physics: Condensed Matter. 17, R461-R524 (2005)

14. Frank, B., Gast, A. P., Russell, T. P., Brown, H. R., Hawker, C.: Polymer mobility in thin films. Macromolecules. 29, 6531-6534 (1996)

15. Masson, J. L., Green, P. F.: Viscosity of entangled polystyrene thin film melts: Film thickness dependence. Physical Review E. 65, 031806 (2002)

16. Bodiguel, H., Fretigny, Ch.: Viscoelastic properties of ultrathin polystyrene films. Macromolecules. 40, 7291-7298 (2007)

17. Leveder, T., Landis, S., Chaix, N., Davoust, L.: Thin polymer films viscosity measurements from nanopatterning method. Journal of Vacuum Science and Technology B. 28, 1251-1258 (2010)

18. Rognin, E., Landis, S., Davoust, L.: Viscosity measurements of thin polymer films from reflow of spatially modulated nanoimprinted patterns. Physical Rewiew E. 84, 041805 (2011)

19. Rognin, E., Landis, S., Davoust, L.: Viscoelastic properties measurements of thin polymer films from reflow of nanoimprinted patterns. Journal of Vacuum Science \& Technology B. 30, 011602 (2012)

20. Jones, R. L., Hu, T., Soles, C. L., Lin, E. K., Reano, R. M., Pang, S. W., Casa, D. M.: Real-time shape evolution of nanoimprinted polymer structures during thermal annealing. Nano Letters. 6, 1723-1728 (2006) 
21. Teisseire, J., Revaux, A., Foresti, M., Barthel, E.: Confinement and flow dynamics in thin polymer films for nanoimprint lithography. Applied Physics Letters. 98, 013106 (2011)

22. Gilormini, P., Teyssèdre, H.: On using the leveling of the free surface of a Newtonian fluid to measure viscosity and Navier slip length. Proceedings of the Royal Society A. 469, 20130457 (2013)

23. Cox, W. P., Merz, E. H.: Correlation of dynamic and steady flow viscosities. Journal of Polymer Science. 28, 619-622 (1958)

24. Carreau, PJ.: Rheological equations from molecular network theories. Transactions of the Society of Rheology. 16, 99 (1972)

25. Williams, M. L., Landel, R. F., Ferry, J. D.: The temperature dependence of relaxation mechanisms in amorphous polymers and other glass-forming liquids. Journal of the American Chemical Society. 77, 3701-3707 (1955)

26. Bicerano, J.: Prediction of polymer properties. CRC Press. (2002)

27. Ro, H. W., Ding, Y., Lee, H. J., Hines, D. R., Jones, R. L., Lin, E. K., Soles, C. L.: Evidence for internal stresses induced by nanoimprint lithography. Journal of Vacuum Science \& Technology B. 24, 2973-2978 (2006)

28. Papenheim, M., Dhima, K., Wang, S., Steinberg, C., Scheer, H. C., Saupe, J., Grimm, J.: Effect of residual stress on replication fidelity with nanoimprint. Journal of Vacuum Science \& Technology B. 32, 06FG01 (2014)

29. Rowland, H. D., King, W. P., Cross, G. L., Pethica, J. B.: Measuring glassy and viscoelastic polymer flow in molecular-scale gaps using a flat punch mechanical probe. ACS nano. 2, 419-428 (2008)

30. Martínez, M. A., Cueto, E., Doblaré, M., Chinesta, F.: Natural element meshless simulation of flows involving short fiber suspensions. J. Non-Newtonian Fluid Mech. 115, 51-78 (2003)

31. González, D., Cueto, E., Chinesta, F., Doblaré, M.: A natural element updated Lagrangian strategy for free-surface fluid dynamics. J. Comput. Phys. $223127-$ $150(2007)$

32. Teyssèdre, H., Gilormini, P.: Extension of the natural element method to surface tension and wettability for the simulation of polymer flows at the micro and nano scales. Journal of Non-Newtonian Fluid Mechanics. 200, 9-16 (2013)

33. Orchard, S. E.: On surface levelling in viscous liquids and gels. Applied Science Research. 11, 451-464 (1962)

34. Henle, M. L., Levine, A. J.: Capillary wave dynamics on supported viscoelastic films: single and double layers. Physical Review E. 75, 021604 (2007) 


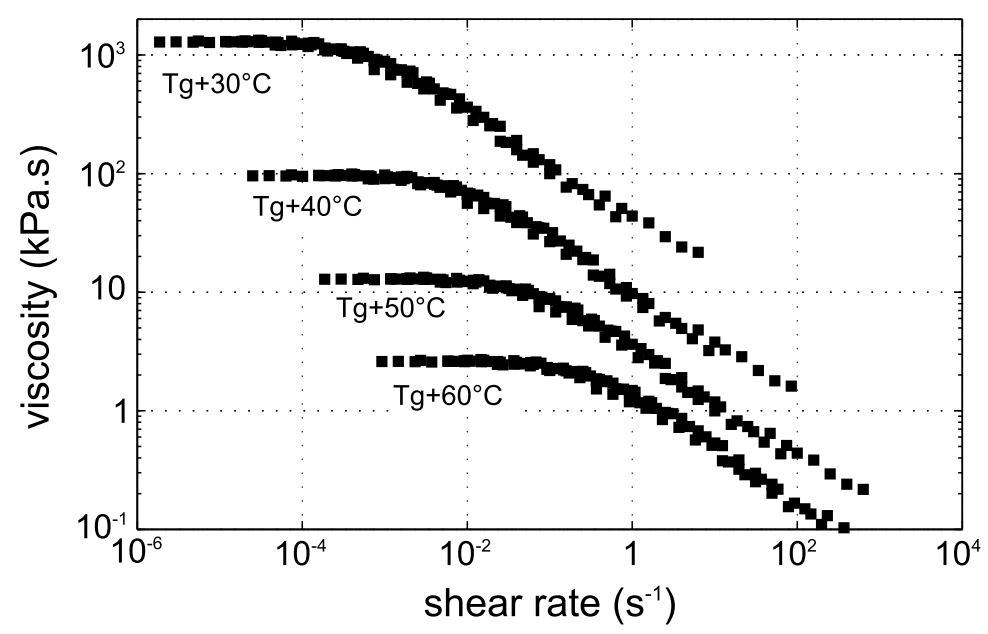

Fig. 1. Evolution of the PS35 viscosity versus shear rate for temperatures over $T_{g}=$ $60^{\circ} \mathrm{C}$.

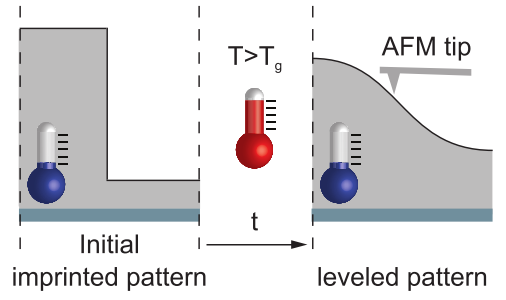

Fig. 2. Illustration of the main steps of the leveling process. An initial pattern is printed in the polymer and its amplitude and pitch are measured by AFM. The film is then heated at a definite temperature above $T_{g}$ during a definite time. Then the film is rapidly cooled down and the reflowed profile is measured by AFM again. 


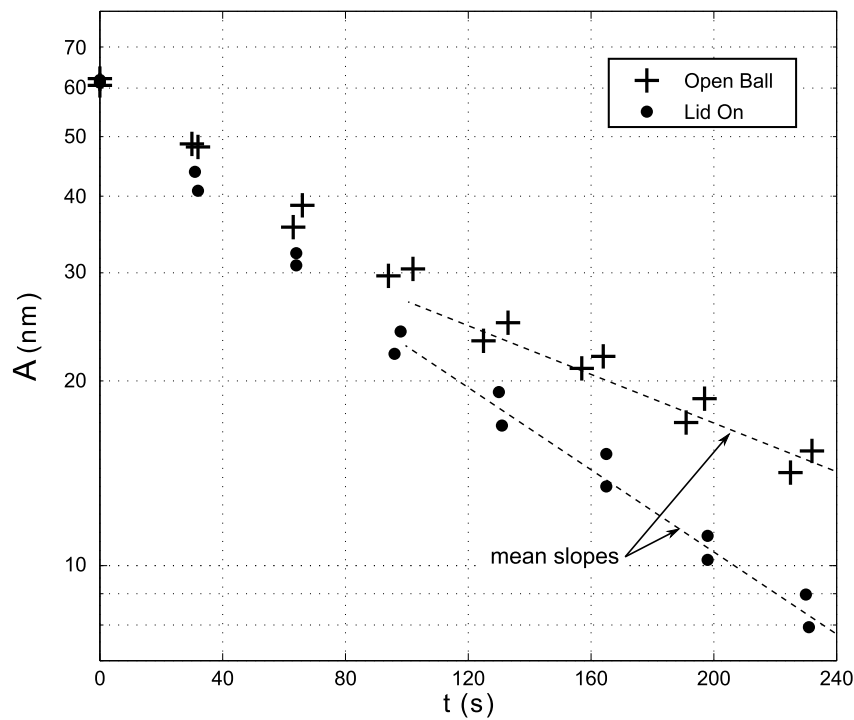

Fig. 3. Evolution of the gratings amplitudes during the leveling process. Experimental data are presented for samples submited to air convection (crosses) and samples covered by a lid to reduce heat exchange (dots). The mean slope of decrease at long times are presented in dashed lines. The experiments have been performed on a hotplate at $100^{\circ} \mathrm{C}$. 


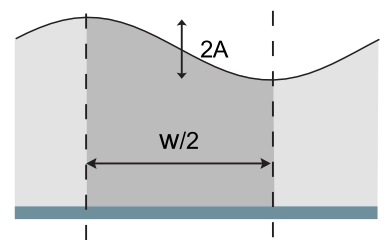

a.i) initial sinusoidal profile

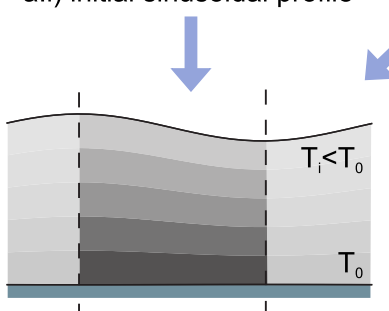

b) partially leveled profile

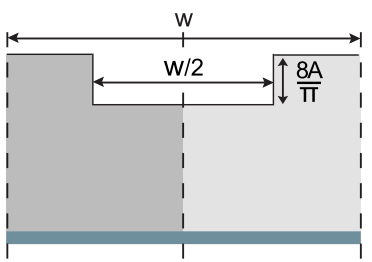

a.ii) initial square wave profile

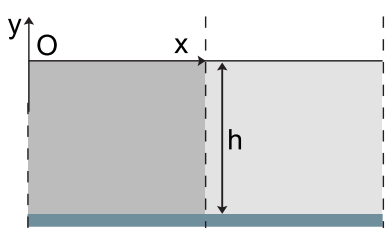

c) equilibrium state thermal gradient in the thickness

Fig. 4. Notations used for the geometry of the problem considered, either with an initial sinusoidal free surface (a.i) or with a square wave one (a.ii), both having the same wavelength $w$. The leveling is performed with unequal imposed temperatures on the substrate and free surface (b). The free surface is levelled until the final equilibrium (c) is reached. The shading illustrates iso-values of temperature. For symmetry reasons (dashed lines define symmetry axes), the problem reduces to the dark gray area. 


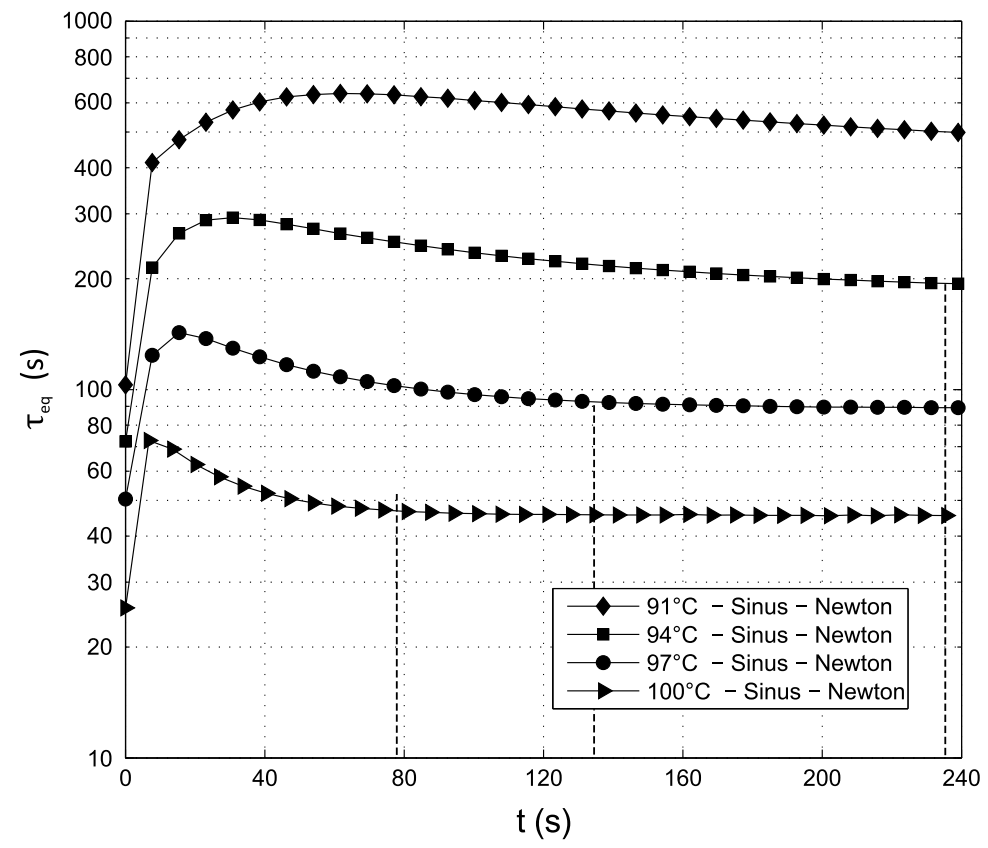

Fig. 5. Evolution of the equivalent relaxing time $\tau_{e q}$ versus time for a free surface temperature ranging from 91 to $100^{\circ} \mathrm{C}$. The temperature of the substrate is determined by equation (5). The simulations are performed with an initial sinusoidal profile and are restricted to the Newtonian behavior.

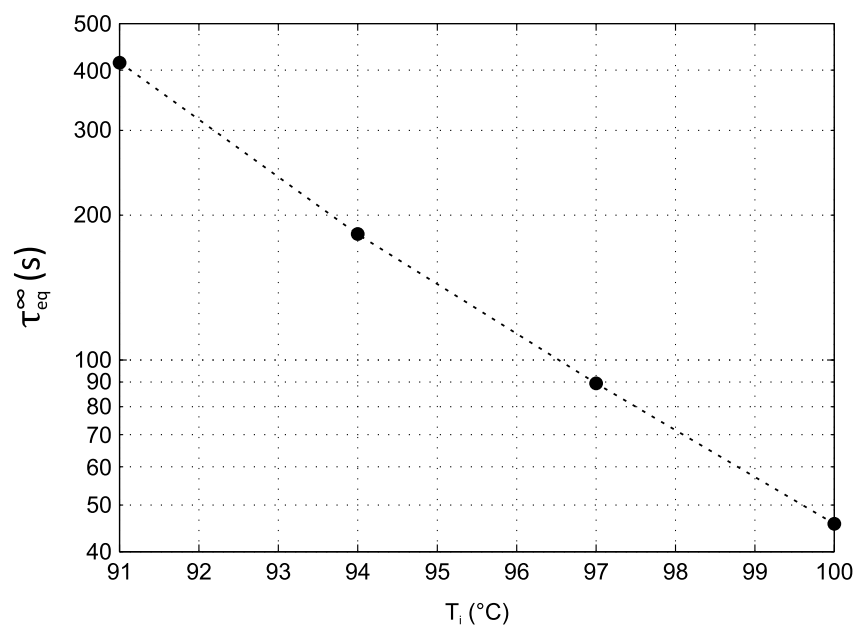

Fig. 6. Evolution of the plateau value $\tau_{e q}^{\infty}$ of the equivalent relaxing time computed numerically versus the free surface temperature $T_{i}$. 


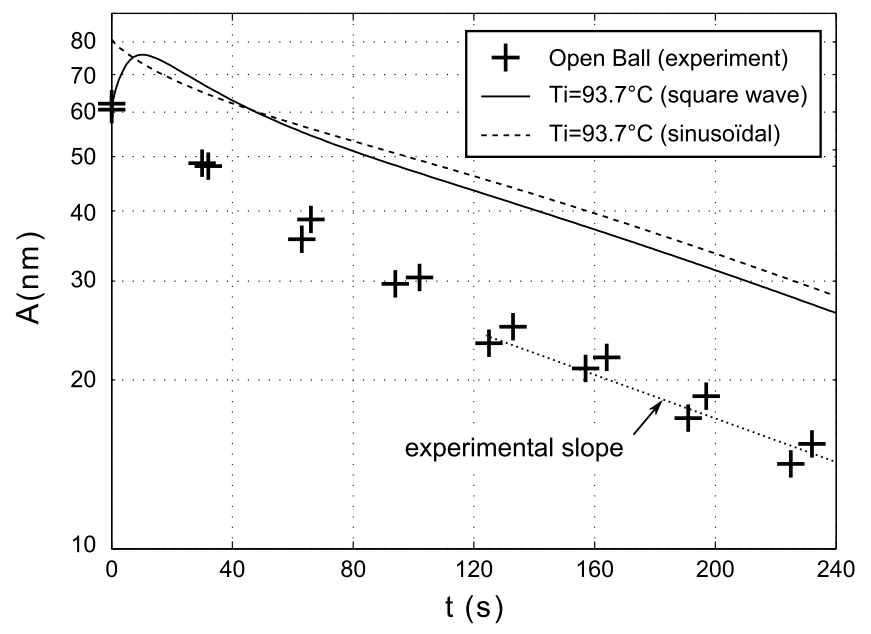

Fig. 7. Comparison between experimental data in the Open Ball case (crosses) and simulated profiles for $T_{i}=93.7^{\circ} \mathrm{C}$. Simulations with square wave (continuous line) and sinusoidal (dashed line) initial profiles are investigated in the case of a Newtionian behavior.

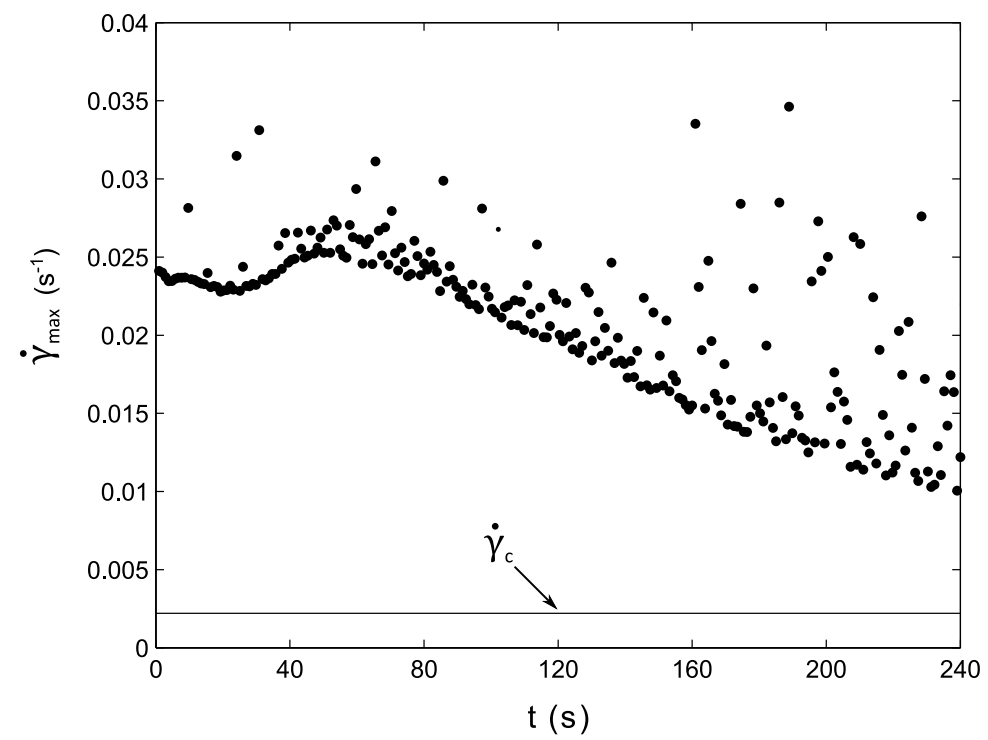

Fig. 8. Evolution of the maximal shear rate $\dot{\gamma}^{\max }$ within the polymer versus time for the main Fourier mode. The critical shear rate $\dot{\gamma}_{c}$ at $94^{\circ} \mathrm{C}$ is plotted in continuous line. Shear-thinning is expected when $\dot{\gamma}^{\max }>\dot{\gamma}_{c}$. 


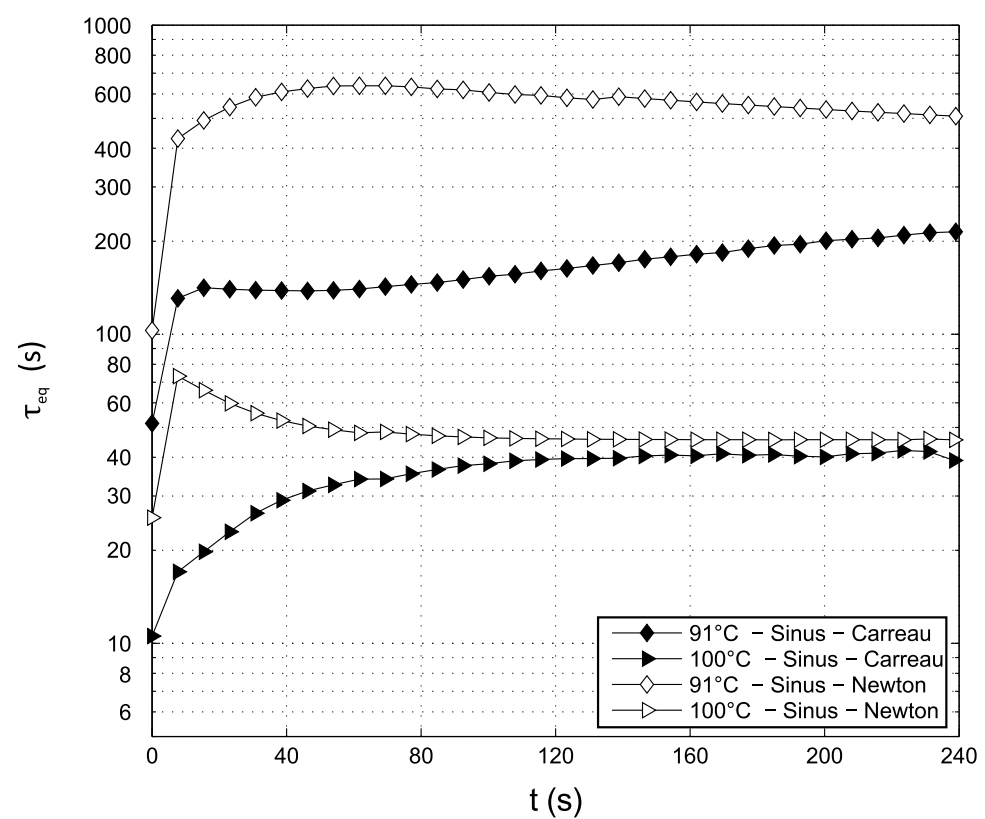

Fig. 9. Evolution of the equivalent relaxing time $\tau$ versus time for a free surface temperature ranging from 91 to $100^{\circ} \mathrm{C}$. The temperature of the substrate is fixed by equation (5). The simulations are performed with an initial sinusoidal profile and a shear-thinning behavior (filled symbols). The equivalent Newtonian case are reported for $T_{i}=100$ and $91^{\circ} \mathrm{C}$ to allow comparison (open symbols). 


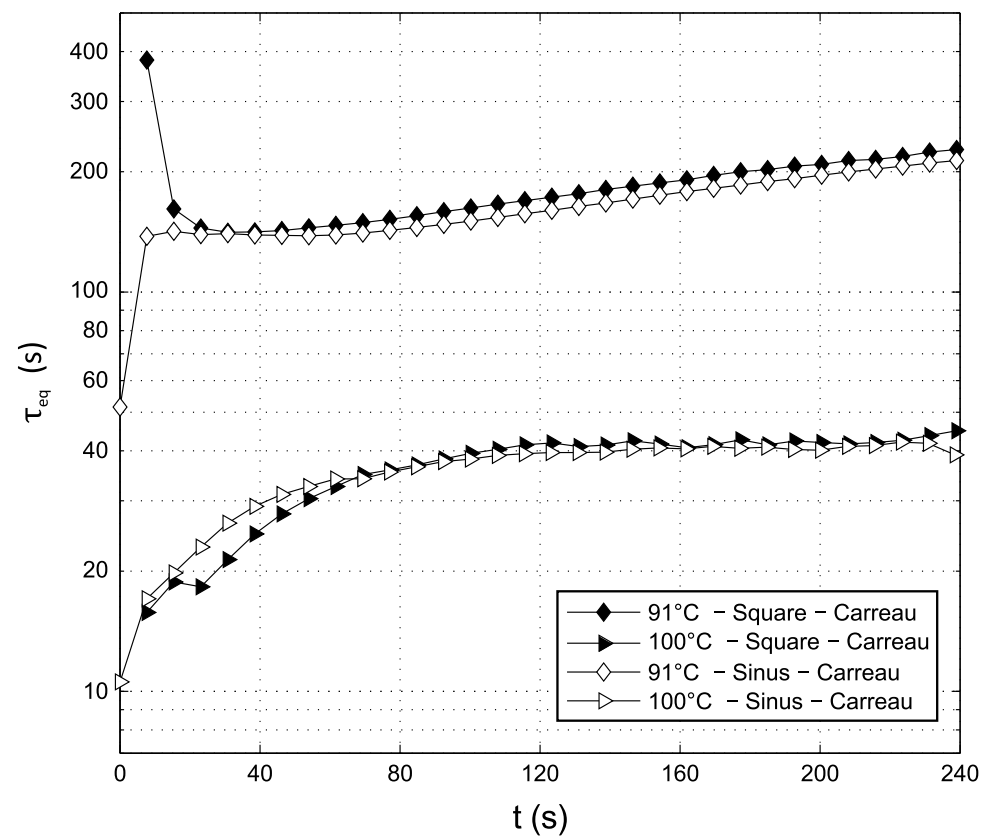

Fig. 10. Evolution of the equivalent relaxing time $\tau$ versus time for a free surface temperature ranging from 91 to $100^{\circ} \mathrm{C}$. The temperature of the substrate is fixed by equation (5). The simulations are performed with an initial square wave profile and a shear-thinning behavior (filled symbols). The equivalent sinusoidal case are reported for $T_{i}=100$ and $91^{\circ} \mathrm{C}$ to allow comparison (open symbols). 


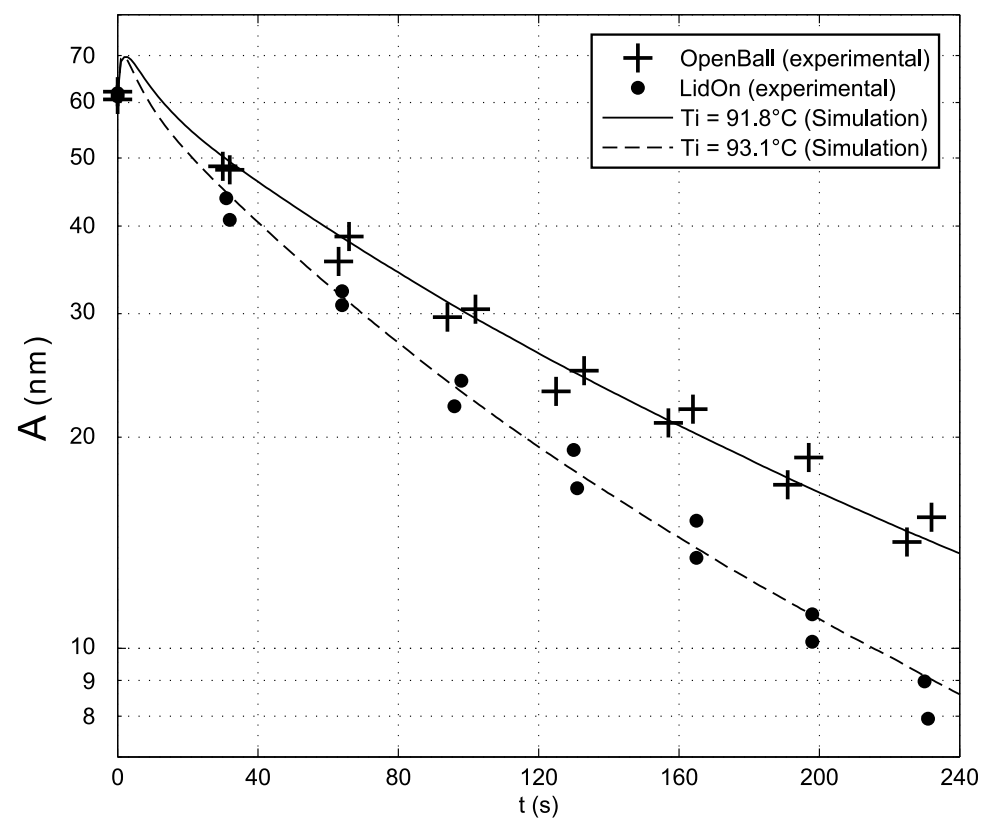

Fig. 11. Experimental data compared to simulations using tuned free surface temperatures and a shear thinning behavior obtained from bulk measurements. A square wave initial profile is used in the simulations. 